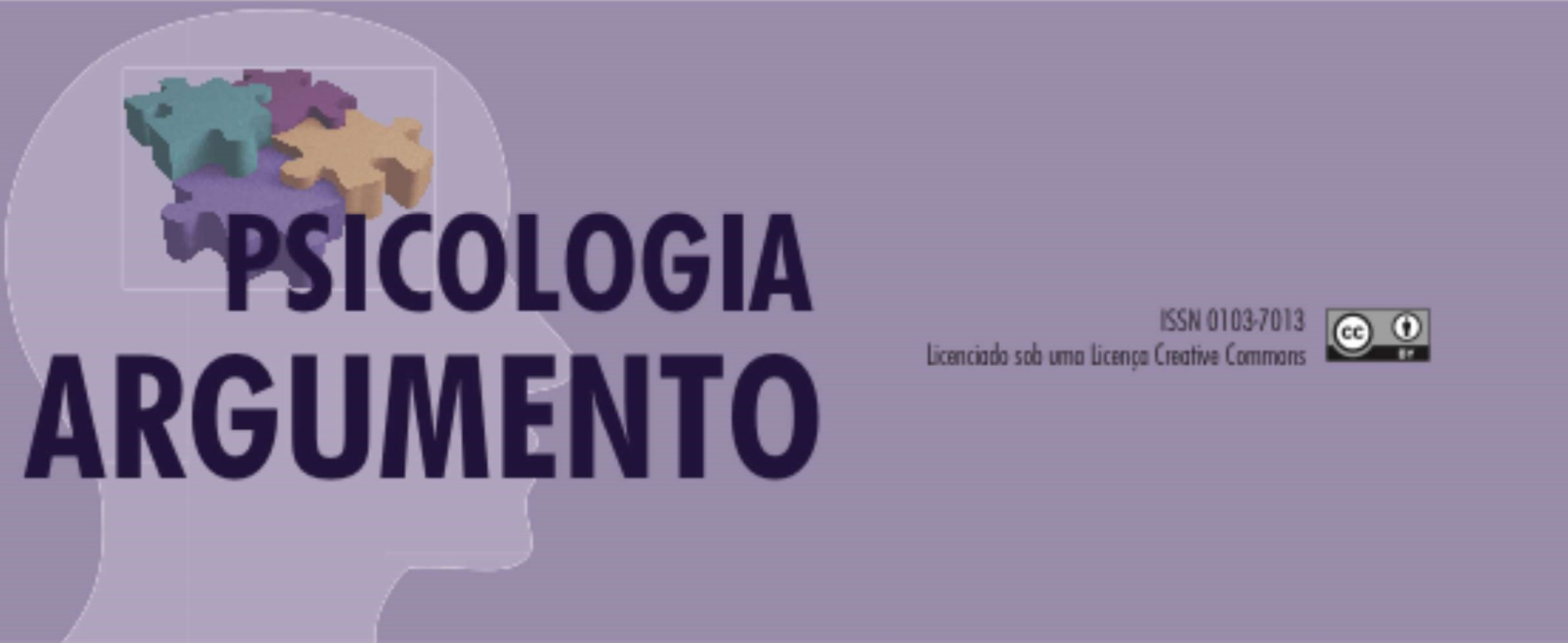

doi: http://dx.doi.org/10.7213/psicolargum. 39.104.A003

\title{
Sentidos de viver com depressão na velhice
}

Meanings of living with depression in old age

Sentidos de vivir com depresión em la vejez

\begin{abstract}
Katia Carreira Pfutzenreuter
Hospital Municipal do Idoso Zilda Arns - Curitiba/PR; katia.pfu@hotmail.com

Itala Villaça Duarte

Hospital Municipal do Idoso Zilda Arns - Curitiba/PR; itala.duarte@hotmail.com

Regina Celia Celebrone

Hospital Municipal do Idoso Zilda Arns - Curitiba/PR; regcelebrone@gmail.com
\end{abstract}

\begin{abstract}
Resumo
Este artigo tem por objetivo analisar os sentidos atribuídos à vivência da depressão por idosos. Trata-se de campo, do tipo transversal e de abordagem qualitativa. Para a caracterização dos participantes foi utilizado um questionário sociodemográfico e para a coleta de dados foi utilizada a Entrevista Narrativa de Doença - McGill Illness Narrative Interview (MINI), entrevista semiestruturada, traduzida, adaptada e validada para o Brasil. Foram analisadas 8 narrativas segundo a metodologia Análise de Conteúdo (Bardin, 2011), e foram sistematizadas as seguintes categorias: (I) A depressão atrelada aos sentidos sociais, agrupando as narrativas que apontam o estigma deste adoecimento psíquico, sendo principalmente relacionando com a loucura; (II) Sentimentos vinculados a depressão e suas repercussões nos laços sociais, incluindo falas sobre a irritabilidade, desânimo e inibição, e necessidade do reconhecimento do outro em relação ao seu sofrimento; e (III) Depressão associada às perdas e lutos de uma vida, havendo correlação entre a depressão na velhice como um acúmulo sucessivas perdas familiares. Os sentidos atrelados a depressão na velhice identificada nessa pesquisa envolvem a dificuldade de nomeação desse
\end{abstract}


sofrimento para além do diagnóstico psiquiátrico e ressalta a necessidade de elaboração de lutos e amparo subjetivo.

Palavras-chave: depressão; psicologia; envelhecimento.

\begin{abstract}
This article aims to analyze the meanings attributed by the elderly to the experience of depression. This is a field research, cross-sectional field study and with a qualitative approach. For the characterization of the participants, a sociodemographic questionnaire was used and for data collection, the Narrative Interview of Disease - McGill Illness Narrative Interview (MINI), semistructured interview, translated, adapted and validated for Brazil was used. Eight narratives were analyzed according to the Content Analysis methodology (Bardin, 2011), and the following categories were systematized: (I) Depression linked to social senses, grouping the narratives that point out the stigma of this psychic illness, being mainly related to madness ; (II) Feelings linked to depression and their repercussions on social ties, including statements about irritability, discouragement and inhibition, and the need to recognize the other in relation to their suffering; and (III) Depression associated with the losses and mourning of a lifetime, with an association between depression in old age as an accumulation of successive family losses. The meanings linked to depression in old age identified in this research involve the difficulty of naming this suffering beyond the psychiatric diagnosis and emphasizes the need to elaborate grief and subjective support.
\end{abstract}

Keywords: depression; psychology; aging.

\title{
Resumen
}

Este artículo tiene como objetivo analizar los significados atribuidos a la experiencia de la depresión en los ancianos. Esta es una investigación de campo, transversal y con un enfoque cualitativo. Para la caracterización de los participantes, se empleó un cuestionario sociodemográfico y para la recolección de datos, se utilizó la Entrevista Narrativa de Enfermedad - McGill Illness Narrative Interview (MINI), entrevista semiestructurada, traducida, adaptada y validada para Brasil. Se analizaron ocho narrativas de acuerdo con la metodología de Análisis de contenido (Bardin, 2011), y se sistematizaron las siguientes categorías: (I) Depresión vinculada a los sentidos sociales, agrupando las narrativas que señalan el estigma de esta enfermedad psíquica, principalmente relacionadas con la locura; (II) Sentimientos relacionados con la depresión y sus repercusiones en los lazos sociales, incluidas las declaraciones sobre irritabilidad, desánimo e inhibición, y la necesidad de reconocer al otro en relación con su sufrimiento; y (III) Depresión asociada con las pérdidas y el duelo de toda la vida, con una asociación entre la depresión en la vejez como una acumulación de pérdidas familiares sucesivas. Se considera que el sujeto anciano afectado por la depresión necesita ser escuchado, considerando las posibilidades de dar un nuevo significado al luto y las historias de vida, por la importancia del apoyo subjetivo y social.

Palabras clave: depresión; psicología; envejecimiento.

\section{Introdução}


Com o progresso da medicina e melhores condições de vida e saúde, os dados do Instituto Brasileiro de Geografia e Estatística (IBGE) evidenciam uma maior longevidade da população brasileira (Albuquerque \& Silva, 2015). Em países em desenvolvimento como o Brasil, os idosos configuram aqueles com 60 anos ou mais (Organização Mundial da Saúde, 2005), sendo a população idosa a que mais cresce no Brasil, apresentando uma estimativa 41,5 milhões, em 2030, e 73,5 milhões, em 2060 (Borges \& Costa, 2015).

As políticas públicas para a garantia de direitos à essa população estão presentes na Política Nacional do Idoso (Brasil, 1994) e no Estatuto do Idoso (Brasil, 2003), dada a necessidade de um amparo civil diferenciado, ponderando os aspectos da senescência e senilidade. A senescência é conceitualizada como o processo de envelhecimento multifatorial e heterogêneo que envolvem mudanças esperadas no âmbito físico, emocional e social. Porém, quando somadas a alguma doença, caracteriza-se como senilidade, exigindo medidas de intervenções distintas (Brasil, 2006).

A depressão é o transtorno de humor mais frequente entre os idosos (American Psychiatric Association, 2013). Ainda assim, costuma ser subdiagnosticada na velhice por ser erroneamente confundida como uma condição esperada desta fase de vida (Ribeiro et al, 2018). O diagnóstico psiquiátrico é identificado por critérios descritivos e objetivos, segundo o Manual Diagnóstico e Estatístico de Transtornos Mentais (American Psychiatric Association, 2013) e a Classificação estatística internacional de doenças e problemas de saúde relacionados à saúde (Organização Mundial da Saúde, 2019).

A sintomatologia psiquiátrica da depressão é caracterizada principalmente pelo humor deprimido e acentuada diminuição de interesse ou prazer, somados pelos seguintes sintomas: perda ou ganho significativo de peso, insônia ou hipersonia, agitação ou retardo psicomotor, fadiga ou perda de energia, sentimentos de inutilidade ou culpa, capacidade diminuída para pensar ou se concentrar e/ou pensamentos recorrentes de morte, em um determinado período (American Psychiatric Association, 2013).

A depressão se apresenta como um recurso simbólico importante na atualidade, que envolve uma variedade de significações culturais. Sendo um fenômeno multifatorial, a depressão necessita ser abordada por diversas visões epistemológicas, a fim de considerar novas formas de compreensão e tratamento (Heguedusch, 2017). 
A psicanálise adota uma posição crítica frente à perspectiva psiquiátrica que releva à segundo plano, a dimensão subjetiva e social no engendramento do sofrimento psíquico (Campos, 2016). A depressão não configura uma doença ou estrutura clínica, pois o diagnóstico psicanalítico é concebido a partir do funcionamento psíquico do sujeito e não pela classificação de sintomas (Borges \& Costa, 2018).

Ainda que a depressão não seja uma estrutura ou diagnóstico em psicanálise, é possível localizá-la a partir do conceito de melancolia. Freud (1917/2010) situa a melancolia como um funcionamento psíquico no qual o indivíduo apresenta dificuldade na elaboração de um luto, a partir de uma perda real ou simbólica, pois este, está identificado ao objeto perdido. A depressão se apresenta como um posicionamento subjetivo em que o sujeito se desvia do seu desejo e permanece em função da angústia e sentimento de vazio (Coser, 2003).

As narrativas singulares sobre o sofrimento e os sentidos que envolvem o diagnóstico de depressão são variados e apontam para a necessidade de uma abordagem da subjetividade desta temática. Dessa forma, objetiva-se analisar os sentidos de viver com depressão na velhice, como um processo singular, que perpassa histórias de vidas.

\section{Método}

A pesquisa foi realizada em um hospital municipal especializado em saúde do idoso de Curitiba (Paraná), após a aprovação do Comitê de Ética da Secretaria de Saúde de Curitiba sob o número do parecer: 3.271 .401 e seguiu as recomendações éticas da Resolução 466/12 (Brasil, 2012).

Esta é uma pesquisa qualitativa, de campo e do tipo transversal onde foram entrevistados oito idosos(as) com o diagnóstico de depressão, registrado em prontuário por um médico psiquiatra do Sistema Único de Saúde (SUS).

Os participantes foram encaminhados pelo serviço de psicologia e psiquiatria do hospital em que a pesquisa foi realizada, equipe previamente informada sobre os critérios de inclusão e exclusão dos participantes e o caráter voluntário da pesquisa. Destaca-se que as pesquisadoras deste estudo não se enquadravam como psicólogas assistentes destes pacientes, portanto, não infligindo nenhum viés ético. 
Aqueles que não estavam aptos a participar por apresentar alterações cognitivas, dificuldade de comunicação verbal, emocionalmente instáveis, quando não estavam cientes sobre o seu diagnóstico de depressão ou que por algum motivo, se recusaram a participar da pesquisa.

As entrevistas foram realizadas individualmente, mediante a leitura e o preenchimento do Termo de Consentimento Livre Esclarecido (TCLE). Foi utilizado um questionário sociodemográfico elaborado para a pesquisa e a Entrevista Narrativa de Doença - McGill Illness Narrative Interview (MINI), um questionário semiestruturado traduzido e adaptado para o Brasil (Leal, Souza, Serpa, Octavio, Oliveira, Dahl, Figueiredo, Salem \& Groleau, 2016). Por se tratar de um instrumento qualitativo, este apresenta o objetivo de gerar narrativas de doença em pesquisa em saúde, considerando seus contextos sócio-históricos (Groleau et al, 2006).

A tradução e adaptação transcultural do questionário para o Português foi realizada por pesquisadores do Laboratório de Estudos em Psicopatologia e Subjetividade do Instituto de Psiquiatria da UFRJ, garantindo a sua aplicabilidade no Brasil com segurança para populações com sintomas psiquiátricos e problemas físicos (Leal, et al, 2016).

Considerando o objetivo desta pesquisa, foram utilizadas as seções I, II, III e V do questionário. A primeira parte propõe que o narrador conte sua própria história de seu modo particular; já a segunda aborda as experiências prototípicas de si e dos outros; seguidamente são questionados os tipos causais de racionalização a partir do contexto sociocultural do entrevistado; e a última seção configura-se sobre o impacto da doença na sua vida e identidade. A seção (IV) "Serviços e Respostas ao Tratamento" é optativa (Groleau, Young \& Kirmayer, 2006) e foi retirada por não se enquadrar nos objetivos deste estudo.

Após a transcrição das gravações, as entrevistas foram submetidas a Análise de Conteúdo proposta por Bardin (2011). Esta metodologia oferta um procedimento estruturado para se obter dados qualitativos. São preconizadas três fases de análise: (1) pré-exploração do material: na qual é realizada a leitura de todo o material, no intuito de identificar sentimentos, significados e impressões sobre os dados coletados, averiguando possibilidades de exploração e interpretações futuras; (2) exploração do material: ocorre 
o estabelecimento das unidades de análises por meio da frequência e relevância do conteúdo, sendo orientada pelos objetivos de pesquisa; (3) no tratamento dos resultados, ocorre a inferência e a interpretação, havendo articulação teórica do pesquisador (Bardin, 2011). Os pesquisados foram codificados por abreviações numéricas (P1-P2...) e as unidades hospitalares dos participantes: enfermaria hospitalar ou ambulatório, a fim de preservar a não identificação deles.

\section{Resultados}

A amostra foi composta por oito indivíduos, sendo quatro do gênero masculino e quatro do feminino e a média de idade atingiu 64 anos (Desvio Padrão: $\pm 3,2$ ). As características sociodemográficas são apresentadas na tabela seguir.

Tabela I - Características sociodemográficas dos participantes de pesquisa. Curitiba, PR, Brasil, 2019.

\begin{tabular}{|c|c|}
\hline Características sociodemográficas & Número de participantes $(\mathrm{N}=8)$ \\
\hline \multicolumn{2}{|l|}{ Escolaridade } \\
\hline Segundo grau completo & 1 \\
\hline Segundo grau incompleto & 1 \\
\hline Fundamental completo & 3 \\
\hline Fundamental incompleto & 3 \\
\hline \multicolumn{2}{|l|}{ Estado civil } \\
\hline Solteiro(a) & 1 \\
\hline Casado(a) & 2 \\
\hline Divorciado(a) & 1 \\
\hline Viúvo(a) & 4 \\
\hline \multicolumn{2}{|l|}{ Atividade econômica } \\
\hline Aposentadoria & 4 \\
\hline Aposentadoria por invalidez & 2 \\
\hline Bolsa família & 1 \\
\hline Pensão por morte & 1 \\
\hline \multicolumn{2}{|l|}{ Religião } \\
\hline Ateu (Ateia) & 1 \\
\hline Católico(a) & 3 \\
\hline Cristão(ã) & 1 \\
\hline Evangélico(a) & 3 \\
\hline
\end{tabular}




\begin{tabular}{ll}
\hline Reside & 1 \\
Sozinho(a) & 7 \\
Com familiares & \\
\hline
\end{tabular}

Dos dados sociodemográficos destaca-se que nenhum participante realizava atividade remunerada ou ocupação econômica, e atribuíram a depressão como um fator emocional que dificultava a realização de atividades laborais, paradoxalmente a falta de ocupação, também influenciava na intensificação do quadro depressivo. Além disso, a aposentadoria por invalidez da participante (P5, 62 anos) foi justificada a partir do diagnóstico de depressão.

As narrativas foram sistematizados em três categorias de análise: (I) A depressão atrelada aos sentidos sociais; (II) Sentimentos vinculados à depressão e suas repercussões nos laços sociais; e (III) Depressão associada às perdas e lutos de uma vida.

\section{Discussão}

\section{A depressão atrelada aos sentidos sociais}

As concepções sociais sobre normalidade e loucura influenciaram no entendimento sobre a depressão, conforme os relatos a seguir: "pela psiquiatra eu tenho distúrbio mental, mas eu nunca pulei em ninguém 'pra' ter distúrbio mental. Transtorno mental não é distúrbio! Aí, peguei e joguei tudo fora os papeis da psiquiatra, 'tô' louca, então não vou precisar mais” (P8, 64 anos); “Antes [da depressão] eu era normal. Eu era calmo, conversava com todo mundo, alegre, 'brincava tudo'... agora 'pra' já não tem mais isso" (P5, 62 anos).

O diagnóstico psiquiátrico foi apresentado como parte da identidade deste idosos e isto pode repercutir no reconhecimento do próprio sofrimento. A dimensão classificatória influencia aprioristicamente no modo de se reconhecer e impacta na consideração de si nas mudanças durante a vida (Heguedusch, 2017). O "ser deprimido" prevaleceu em oposição ao "estar depressivo", o que denota o entendimento de uma condição crônica e estável.

Para todos os sujeitos da pesquisa, o diagnóstico de depressão foi realizado em um período anterior a velhice, apresentando um sofrimento de longa duração e difícil 
superação. A depressão foi caracterizada como um traço de identidade, permeado por momentos de crises, utilizaram termos como: "cair na depressão" (P1, 71 anos); "atacou a depressão" (P2, 64 anos); "se entregar à depressão” (P6, 60 anos).

A concepção de normalidade também foi abordada a partir de concepções religiosas, exemplificado nas seguintes falas: "vejo o pastor falar que depressão não é de Deus, mas só a gente que passa por essas coisas que sabe, né?” (P8, 64 anos); “Tem pessoas que diz assim, que não é coisa normal, tem gente fala até besteira, 'isso é coisa do diabo' não sei o que é, mas é coisa da vida da gente ne, eu acho...” (P3, 66 anos). A normalidade, enquanto uma visão objetiva, é questionável por apresentar uma concepção rígida e que nega a subjetividade da experiência (Canguilhem, 2011).

Os participantes identificaram o impasse entre a vivencia e a compreensão de normalidade advinda do discurso social, causando efeitos e repercussões singulares. Questionaram sobre os seus sentimentos e a difícil representação dessa vivência por meio das palavras. A nomeação do sofrimento por meio de um diagnóstico presume critérios pré-determinados e marca o sujeito no discurso médico, podendo ocasionar uma dificuldade na ampliação de sentidos. A linguagem atende à uma tentativa de nomeação, ainda que sempre incompleta, é por meio dela que se experimenta dizer aquilo que escapa a significação (Mucida, 2012).

\section{Sentimentos vinculados a depressão e suas repercussões nos laços sociais}

Os principais sentimentos atrelados à vivência da depressão foram de irritabilidade e desânimo, os quais costumam envolver conflitos interpessoais e isolamento social. A irritabilidade foi referida pelos participantes como um sentimento incontrolável e sem uma causa especifica, mas envolvem preocupações e pensamentos incessantes. Este fato pode ser exemplificado pelas falas: “A depressão é as coisas que não dão certo, eu fico nervoso, as coisas começo bater, qualquer coisa já quero brigar com os outros, fico nervoso... estressado demais, acho que pra mim isso daí, não é normal” (P5, 62 anos); “Não sei dizer, não sei explicar isso 'dai'... depressão 'pra' mim acho que é pessoa que pensa muita coisa, eu acho que não é certo, não é normal querer brigar, quebrar tudo... eu acho que não é normal, né? 'pra' mim acho que não é” (P5, 62 anos). 
Diante deste sentimento incontrolável de irritabilidade, o isolamento social se apresenta como uma alternativa para lidar com seus sentimentos e comportamentos impulsivos. Ainda que este possa ser uma saída, Os idosos indicaram que a reclusão é uma condição difícil de ser superada, conforme as seguintes falas: "O melhor tratamento para depressão é o sossego” (P2, 64 anos); “Quero sair e não dá, não tenho nem vontade de sair de casa!" (P5, 62 anos); "É uma doença que deixa você de mãos atadas, não tem ânimo, não dorme, não come." (P8, 64 anos).

O desejo de retomar atividades prazerosas foi mencionado por todos e em especial as atividades que pudessem "ocupar a cabeça", trazendo novos sentidos e experiências de vida: "a gente preenchia o tempo com aquilo [fazer desenho e jogar dominó] (...) a cabeça da gente preenchia com aquilo (...) Quando a gente fica bem fraco, vai pra igreja” (P7, 65 anos); "lá [na igreja] a gente escuta coisas de Deus, alivia a cabeça da gente" (P8, 64 anos).

O apoio familiar foi considerado importante pelos idosos, ainda que essa abordagem frequentemente pudesse gerar sentimentos de frustrações e impotência, por envolver estereótipos, conforme narrado a seguir: "chegam 'pra' mim: 'você tem que se animar, tem que reagir', mas não é bem assim a depressão, eu não sei bem explicar..." (P2, 64 anos); “Olham com pena, tem gente que pensa que é 'sem vergonhice', outros pensam que tá 'se fazendo'”, (P8, 64 anos); “Ficam com dó da gente, vivem perguntando 'tá melhor?' (...) acho que eles queriam ajudar, mas atrapalham, (...) não é fácil assim se levantar" (P2, 64 anos).

A escuta qualificada do psicólogo foi considerada fundamental, sendo que metade dos participantes realizavam acompanhamento psicológico de grupo na instituição da pesquisa. O grupo psicoterapêutico foi mencionado como um meio de trabalhar aspectos emocionais da depressão e a possibilidade de trocar experiencias. No trabalho das subjetividades em grupo, é possível o reconhecimento de si pelo olhar do outro; as interações dialógicas sociais em um grupo operativo psicanaliticamente orientado, oportunizam que idosos possam realizar (re)negociações com as suas próprias subjetividades e de outrem (Celebrone, 2019).

Já o relacionamento médico-paciente foi percebido como um relacionamento impessoal, o que dificulta o tratamento, além da medicação psiquiátrica apresentar 
impasses, como a: dificuldade na adaptação medicamentosa, medo de dependência, demasiados efeitos colaterais e a grande quantidade de ingestão de remédios.

\section{Depressão associada às perdas e lutos de uma vida}

Os idosos desse estudo relacionaram o início da depressão com o falecimento familiar e da recorrência de perdas conseguintes. Isto pode ser verificado nas falas a seguir: "Acho que a depressão é na 'perca' das coisas, vai perdendo a família e vai ficando assim...” (P3, 66 anos); “Começou com as perdas, foi indo, foi indo, um atrás do outro já né?" (P4, 63 anos); "minha mãe faleceu, meu filho faleceu, 'dai'foram falecendo (...), parece que eu não tenho animo 'pra' mais nada...” (P8, 64 anos). Neste momento de vida, é comum haver uma maior recorrência de falecimentos de pessoas próximas e um balanço sobre perdas e ganhos ocorridos durante a vida.

$\mathrm{Na}$ velhice ocorre a perda de objetos investidos psiquicamente por anos, como atividade laboral e relacionamentos, o que pode tornar o processo de luto mais penoso (Cherix \& Junior, 2018). O luto é um processo de elaboração frente à perda de um objeto real ou ideal, ou seja, é uma reorganização psíquica para realizar uma substituição, ainda que parcial (Freud, 1917/2010).

Os idosos ilustraram a existência de pensamentos recorrentes sobre as suas perdas, sendo este um modo de persistir com o que na realidade já se perdeu: "tem todos esses passados, deito e fico lembrando (...) Nós éramos em 6 irmãos, ficou o eu, o mais velho passado que não esqueço" (P5, 65 anos); "minha cabeça que é da depressão, a minha cabeça vive pesada e quando tenho problema ataca" (P2, 64 anos). A reminiscência se apresenta como um importante organizador psíquico, que tem como propósito manter o passado vivo (Goldfarb, 2004).

$\mathrm{Na}$ contemporaneidade, o idoso enlutado encontram dificuldades de ser amparo pelo social de forma satisfatória, na qual ele possa promover novos investimentos libidinais, bem como não encontra a imagem de uma velhice positiva e valorosa (Cherix \& Junior, 2018). Essa realidade pode mudar pela crescente visibilidade das demandas sociais e psicológicas da população idosa, havendo a necessidade da criação de novos espaços sociais para debates. Destaca-se que a amostra desta pesquisa faz parte de uma população economicamente desfavorecida, o que implica a necessidade de medidas sociais inclusivas e acessíveis. O Ministério da Saúde (2018) divulgou em seu portal o 
"Estudo longitudinal da saúde dos idosos brasileiros" (Elsi-Brasil, 2019), o qual apontou que $75,3 \%$ dos idosos brasileiros dependem exclusivamente dos serviços prestados no Sistema Único de Saúde.

O sistema de saúde possui um papel importante no tratamento holístico do idoso adoecido pela depressão, bem como na implementação de práticas preventivas que incentivem a qualidade de vida e inclusão social do idoso na sociedade. A importância do amparo psicológico é reforçada pela psicanalista Délia Goldfarb (2004), que vincula a impossibilidade de luto com o desencadeamento do quadro demencial. Este, como um modo de defesa psíquica, frente às perdas significativas não elaboradas.

Ademais, a ideação suicida foi identificada por metade dos entrevistados e um destes estava internado por intoxicação exógena. Frente a este sofrimento psíquico, a depressão é um dos principais fatores de risco para o suicídio, que vem aumentando consideravelmente na população idosa, assim como: as relações sociofamiliares fragilizadas, o isolamento social, a solidão, o tempo de vida ocioso e tedioso, o uso abusivo do álcool e a percepção negativa do envelhecer (Minayo, Teixeira \& Martins, 2016).

A prevenção do suicídio na população idosa envolve a conscientização de familiares e profissionais com vistas à possibilitar o envelhecimento ativo (Gomes, Oliveira, Melos, Silva, Fernandes \& Oliveira, 2019). O amparo social necessita ser qualificado e o debate sobre essa temática desmistificado, pois o diagnóstico de depressão nesta fase, deve considerar aspectos individuais e sociais do idoso.

A psicanálise aposta na possibilidade do sujeito ressignificar sua história de vida por meio do discurso, um trabalho que pela via inconsciente, possibilita retificações subjetivas, em que sujeito é convocado a implicar-se no próprio sofrimento, na sua história de vida e no seu desejo (Silva, 2018).

\section{Considerações finais}

Os idosos desta pesquisa conceberam a depressão como um sofrimento difícil de ser simbolizado e elaborado em palavras. Vincularam a depressão ao discurso social de loucura, o que inscreve subjetivamente os seus modos de vivenciarem este adoecimento. 
Frente à quase inexistência de lugares sociais de fala e de escuta dos sofrimentos psíquicos dos idosos é que a clínica psicanalítica desponta como oportunidade e possibilidade de elaboração de afetos, em palavras, que evitam as instaurações de quadros demenciais e depressivos.

É comum a velhice ser um momento de vida marcado por perdas recorrentes, consequentemente o convívio social de idosos pode ser mais restrito. Com a crescente visibilidade às demandas dos idosos, novos espaços sociais e atividades significativas estão sendo propostas, possibilitando atividades prazerosas, projetos de vida e afetos. Este estudo destaca o reconhecimento da subjetividade do idoso, que muitas vezes é abordada do ponto de vista normativo e ainda silenciada. Os sentidos atrelados a depressão envolveram uma dificuldade de nomeação desse sofrimento para além do diagnóstico psiquiátrico e ressalta a necessidade de elaboração de lutos e amparo subjetivo aos idosos, frente às recorrentes perdas.

\section{Referências}

Albuquerque, F. R. P. C. \& Silva, L. G. D. C. (2015). Tendências dos níveis e padrões de mortalidade e seus diferenciais regionais no período 2000-2030: Brasil, Grandes Regiões e Unidades de Federação. Mudança demográfica no Brasil no início do século XXI. Subsídios para as projeções da população. Rio de Janeiro: IBGE. Recuperado de: https://biblioteca.ibge.gov.br/visualizacao/livros/liv93322.pdf

Associação Americana de Psiquiatria.(2013). Manual diagnóstico e estatístico de transtornos mentais (5a. ed.). doi: https://doi.org/10.1176/appi.books.9780890425596.

Bardin, L. (2011). Análise de conteúdo. Lisboa: Edições, 70.

Borges, F. S. \& Costa, P. J. (2018). Um estudo psicanalítico das relações entre depressões neuróticas e desejo: aproximações possíveis. Ágora: Estudos em Teoria Psicanalítica, 21 (3), 343-353. doi: https://dx.doi.org/10.1590/s1516-14982018003006

Borges, G. M., Campos, M. B. \& Silva, L. G. C. (2015) Transição da estrutura etária no Brasil: oportunidades e desafios para a sociedade nas próximas décadas In: Ervatti, L. R. Borges, G. M., Jardim, A. P. (Org.). Mudança demográfica no Brasil no início do século 
XXI: subsídios para as projeções da população. (pp. 138-151). Rio de Janeiro: IBGE. Recuperado de https://biblioteca.ibge.gov.br/visualizacao/livros/liv93322.pdf

BRASIL. Lei n. 10,741, de 1 de outubro de 2003, dispõe sobre o estatuto do idoso e da outras providencias. Diário Oficial da União, Brasília, DF, 3 out. 2003. Recuperado em 20 de junho, 2019, de http://www.planalto.gov.br/ccivil 03/leis/2003/110.741.htm

BRASIL. Ministério da Saúde; Secretaria de Atenção à Saúde; Departamento de Atenção Básica. Envelhecimento e saúde da pessoa idosa. Brasília; 2006. (Cadernos de Atenção $\begin{array}{lllll}\text { Básica, } & \text { n. } & \text { 19). }\end{array}$ http://189.28.128.100/dab/docs/publicacoes/cadernos ab/abcad19.pdf

BRASIL (2012). Ministério da Saúde. Conselho Nacional de saúde. Resolução 466 de 12 de dezembro de 2012. Aprova diretrizes e normas regulamentadoras de pesquisas envolvendo seres humanos. Brasília: Diário Oficial da União. Recuperado em: http://conselho.saude.gov.br/resolucoes/2012/Reso466.pdf

Campos, E. B. V. (2016). Uma perspectiva psicanalítica sobre as depressões na atualidade. Estudos Interdisciplinares em Psicologia,7(2), 22-44. doi: https://dx.doi.org/10.5433/2236-6407.2016v7n2p22

Canguilhem, G. (2011). O normal e o patológico (7a. ed.). Rio de Janeiro: Forense Universitária.

Cherix, K., Junior, C. \& Ernesto, N. (2018). Luto e melancolia nas demências: a psicanálise na clínica do envelhecimento. Trivium - Estudos interdisciplinares, 10(2), 182-195. doi: https://dx.doi.org/10.18379/2176-4891.2018v2p.182

Celebrone, R. C. (2019). Grupo com idosos: lugar de envelhescências. Curitiba: Juruá.

Coser, O. (2003) Depressão: clínica, crítica e ética. Rio de Janeiro: Editora Fiocruz.

Freud, S. (1915/2010). Luto e melancolia. In: Freud S. (1914-1916). Obras completas. Introdução ao narcisismo, ensaios de metapsicologia e outros textos, v.12, (pp. 171194). São Paulo: Companhia das Letras (Original publicado em 1915).

Goldfarb, D. C. (2004) Demências. São Paulo: Casa do Psicólogo 
Groleau, D., Young, A. \& Kirmayer, L. J. (2006). The McGill Illness Narrative Interview (MINI): an interview schedule to elicit meanings and modes of reasoning related to illness experience. Transcultural psychiatry, 43 (4) 671-691. doi: https://doi.org/10.1177/1363461506070796.

Gomes de M. S. E. D., Oliveira L. R. G., Melos dos S. L., da Silva A. M. E. Fernandes de A. L. \& de Oliveira S. J. V. (2019). Suicide between elderly in Brazil: a literature review of the last 10 years. Psicología, Conocimiento y Sociedad, 9(1), 205-220.doi: https://dx.doi.org/10.26864/pcs.v9.n1.12.

Heguedusch, C. V. (2017). Depressão na atualidade: estrutura psíquica ou metáfora do psiquismo? Um diálogo entre Maria Rita Kehl e Pierre Fédida. Cad. Psicanálise (CPRJ), 39, 29-51. Recuperado de:

http://pepsic.bvsalud.org/scielo.php?script=sci_arttext\&pid=S1413$62952017000200002 \& \operatorname{lng}=$ pt\&tlng=pt.

Leal, E. M., Souza, Alicia N. de, Serpa J., Octavio D. de, Oliveira, I. C. de, Dahl, C. M., Figueiredo, A. C., Salem, S., \& Groleau, D. (2016). McGill Entrevista Narrativa de Adoecimento - MINI: tradução e adaptação transcultural para o português. Ciência \& Saúde Coletiva, 21(8), 2393-2402. https://dx.doi.org/10.1590/1413$\underline{81232015218.08612015 .}$

Lima-Costa M. F., de Andrade F.B., Souza PRB, Neri A. L., Duarte Y. A. O., Castro-Costa E \& de Oliveira C. (2018). The Brazilian Longitudinal Study of Aging (ELSI-Brazil): Objectives and Design. Am J Epidemiol.;187(7):1345-1353. doi: 10.1093/aje/kwx387.

Minayo, M. C. D. S., Teixeira, S. M. de O. \& Martins, J.. C. de O. (2016). Tédio enquanto circunstância potencializadora de tentativas de suicídio na velhice. Estudos de Psicologia (Natal), 21(1), 36-45. https://dx.doi.org/10.5935/1678-4669.20160005

Ministério da Saúde (2018, 1 de outubro). "Estudo longitudinal da saúde dos idosos brasileiros". Recuperado em: $\quad$ http://www.saude.gov.br/noticias/agenciasaude/44451-estudo-aponta-que-75-dos-idosos-usam-apenas-o-sus

Organização Mundial da Saúde. (2005). Envelhecimento ativo: uma política de saúde. Tradução Suzana Gontijo. Brasília: Organização Pan-Americana da Saúde-OPAS. 
Recuperado

em:

http://bvsms.saude.gov.br/bvs/publicacoes/envelhecimento ativo.pdf

Organização Mundial da Saúde. (2019). Classificação estatística internacional de doenças e problemas de saúde relacionados ( $11^{\mathrm{a}} \mathrm{ed}$.). Recuperado em: https://icd.who.int/

Perez M., Sirelli N. M. (2015) A medicalização do mal-estar: a escuta psicanalítica como um modo de resistência. Psicanálise \& Barroco em revista, 13(2), 117-13.

Política Nacional do Idoso: Lei 8.842 de 04/01/1994- Brasilia: MPAS, SAS, 1997.

Mucida, A. (2012). O sujeito não envelhece - psicanálise e velhice. 2 ed. Belo Horizonte: Autêntica.

Ribeiro, V. S., Rosa, R. S., Sanches, G. D. J. C., Ribeiro, Í. J. S., \& Casotti, C. A. (2018). Qualidade de vida e depressão em domicílios no contexto doméstico. Revista Enfermería Actual en Costa Rica, (34), 2.

Silva, J. M. D. (2018). A clínica psicanalítica com idosos uma construção. Estudos de Psicanálise, (49), 115-123. Recuperado em: http://pepsic.bvsalud.org/scielo.php?script=sci_arttext\&pid=S0100$34372018000100011 \& \operatorname{lng}=$ pt\&tlng=pt. 